\title{
DEMOCRAZIA, COSTITUZIONE E MUTAMENTO SOCIALE
}

\author{
DEMOCRACY, CONSTITUTION AND SOCIAL CHANGE
}

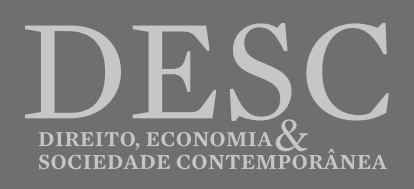




\title{
DEMOCRAZIA, COSTITUZIONE E MUTAMENTO SOCIALE
}

\section{DEMOCRACY, CONSTITUTION AND SOCIAL CHANGE}

\author{
Valentina Pazé \\ Università degli Studi di Torino \\ valentina.paze@unito.it
} www.didattica-cps.unito.it/persone/valentina.paze

\begin{abstract}
Riassunto: Costituzione e mutamento sociale. Rivoluzione come movimento e come mutamento. Diritti sociali. Bilancio pubblico. Democrazia e vincoli “anti-sociali".
\end{abstract}

Parole chiavi: Mutamento sociale. Diritti sociali. Democrazia.

\begin{abstract}
Constitution and social change. Revolution as a movement and as changing. Social rights. Public budget. Democracy and "anti-social” constraints.
\end{abstract}

Keywords: Social change. Social rights. Democracy.

1. In un scritto intitolato La resistenza all'oppressione oggi, Norberto Bobbio scrive: "il processo che diede luogo allo Stato liberale e democratico si può ben chiamare un processo di 'costituzionalizzazione' del diritto di resistenza e di rivoluzione". ${ }^{1}$

In questo passo sono contenute due tesi distinte. Per un verso, riferendosi allo Stato liberale (qui inteso come Stato costituzionale), Bobbio sostiene che rende superfluo l'esercizio del diritto di resistenza contro i sovrani che abusano del loro potere. L" "appello al cielo" di Locke lascia il passo all'“appello ai giudici”, in particolare a quelli della Corte costituzionale, incaricati di vigilare affinché il potere politico non travalichi i limiti ad esso assegnati dalla carta fondamentale, violando i diritti delle persone e dei cittadini. In secondo luogo Bobbio si riferisce allo Stato democratico come a quel regime che legalizza e regola il ricorso alla rivoluzione: "l'istituto del suffragio universale può essere considerato il mezzo attraverso cui avviene la costituzionalizzazione del potere del popolo di rovesciare i governanti, se pure [...] entro i limiti di regole prestabilite, di un potere che prime era riservato esclusivamente al fatto rivoluzionario". ${ }^{2}$

\footnotetext{
1 N. Bobbio, L'età dei diritti, Einaudi, Torino 1990, p. 165.

2 Ivi, p. 167.
} 
Bobbio sembra qui interpretare l'idea di rivoluzione in modo riduttivo, ponendo l'accento sul "rovesciamento dei governanti", senza nulla aggiungere in merito alla radicalità del cambiamento possibile. Altrove, aveva invitato a distinguere due dimensioni dell'idea moderna di rivoluzione, che stanno tra loro in un rapporto di causa-effetto, o di mezzo-fine: il movimento e il mutamento. ${ }^{3}$ Il movimento rivoluzionario si distingue da altri possibili forme di azione collettiva per il suo carattere improvviso, illegale, violento. Si differenzia dunque in particolare dal riformismo, che si propone di trasformare la realtà in modo graduale e progressivo, rimanendo entro i confini della legalità. Il mutamento rivoluzionario si distingue da altri tipi di mutamento essenzialmente per il suo carattere radicale. Non consiste nel semplice ricambio della classe dirigente, e neanche nel solo passaggio da una forma di governo all'altra, ma in una trasformazione che va in profondità, investendo la sfera economico-sociale, oltre a quella politica.

Credo di non forzare il pensiero di Bobbio attribuendogli la tesi che la democrazia - e specificamente la democrazia costituzionale - offre la possibilità di realizzare la rivoluzione come mutamento (o fine), senza il bisogno di ricorrere alla rivoluzione come movimento (o mezzo). Essa offre in particolare ai ceti subalterni un canale per esprimere le loro rivendicazioni e realizzare un progetto di società giusta, senza rompere con la legalità e senza ricorrere alla violenza. Spostando la lotta di classe dalle piazze alle aule parlamentari.

L'interpretazione di Bobbio del rapporto tra democrazia e rivoluzione che ho qui richiamato è diametralmente opposta a quella che è stata proposta da Sheldon Wolin, per il quale la democrazia, una volta istituzionalizzata e racchiusa entro la cornice di regole costituzionali, conduce alla "suppression of revolution". Non alla realizzazione della rivoluzione con altri mezzi, ma alla neutralizzazione, o comunque a un serio infiacchimento, di ogni spinta verso un cambiamento autentico. ${ }^{4}$

Per capire la posizione di Wolin bisogna tenere presente che egli distingue due accezioni tra loro molto diverse di democrazia. In base alla prima, da lui definita "aconstitutional", la democrazia è un "mode of being", una "culture", una "political practise" intrinsecamente "rivoluzionaria", che coincide in ultima analisi con la lotta che i ceti meno abbienti conducono per strappare alle élite il diritto di contare. ${ }^{5}$ Non a caso i critici antichi della democrazia la associavano alla violenza (l'anonimo autore della Athenaion Politeia), alla libertà sfrenata e anarchica (Platone), a qualcosa di informe e concettualmente antitetico a qualsiasi tipo

\footnotetext{
3 N. Bobbio, La rivoluzione come movimento e mutamento, in Teoria generale della politica, a cura di M. Bovero, Einaudi, Torino 1999, pp. 564 e sg.

4 S. Wolin, Norm and Form: The Constitutionalizing of Democracy, in J.P. Euben, J.R. Wallach, J. Ober (eds.), Athenian Political Thought and the Reconstruction of American Democracy, Cornell University Press, Ithaca and London 1994, p. 29

5 Cfr. Wolin 1994: 37: "Instead of a concepcion of democracy as indistinguishable from its constitution, I propose accepting the familiar charges that democracy is inherentely instable, inclined toward anarchy, and identified with revolution and using these traits as the basis for a different, aconstitutional conception of democracy".
} 
di costituzione (Aristotele). Questa concezione sarebbe stata soppiantata fin dal IV secolo ad Atene da un modello di "constitutional democracy" finalizzato a imbrigliare e addomesticare il potere del popolo, percepito come una turba violenta e irrazionale, in nome del primato di una legge immutabile. ${ }^{6}$ Si sarebbe così depotenziata la carica rivoluzionaria della democrazia nel suo significato originario. Anche la versione moderna della democrazia costituzionale altro non sarebbe che "an ideological construction designed not to realize democracy but to reconstitute it and, as a consequence, repress it". ${ }^{7}$

Nel formulare un simile giudizio Wolin pensa in particolare alla Costituzione degli Stati Uniti d'America, da lui giudicata fin dalle origini poco democratica e congegnata per diluire le spinte rivoluzionarie e per impedire a qualsiasi maggioranza di adottare politiche sociali in grado di intaccare i rapporti economici esistenti. Paradigmatiche sarebbero, in questo senso, le resistenze opposte dai giudici della Corte suprema al New Deal in nome della Costituzione e della libertà economica ivi garantita.

Le tesi di Bobbio sono formulate in un contesto molto diverso: quello del costituzionalismo europeo del secondo dopoguerra, nato dalla Resistenza al nazi-fascismo, cui parteciparono attivamente forze che si riconoscevano negli ideali socialisti e comunisti. La Costituzione italiana, in particolare, frutto del compromesso tra le diverse culture rappresentate all'assemblea costituente, ha una forte impronta sociale: l'art. 3 attribuisce alla Repubblica il compito di "rimuovere gli ostacoli di ordine economico e sociale che, limitando di fatto la libertà e l'eguaglianza dei cittadini, impediscono il pieno sviluppo della persona umana e l'effettiva partecipazione di tutti i lavoratori all'organizzazione politica, economica e sociale del Paese"; l'art. 4 riconosce il diritto al lavoro tra i principi fondamentali dell'ordinamento; l'art. 41 garantisce "l'iniziativa economica privata", purché non si svolga “in contrasto con l'utilità sociale o in modo da recare danno alla sicurezza, alla libertà, alla dignità umana". Quanto al diritto di proprietà, di cui si occupa l'art. 42, non è più assoluto e "inviolabile" - come ancora lo proclamava lo Statuto Albertino - ma viene "riconosciuto e garantito dalla legge che ne determina i limiti allo scopo di assicurarne la funzione sociale". Come ha sostenuto Stefano Rodotà, pur rimanendo neutrale rispetto alla scelta di particolari politiche economiche, la Costituzione del 1948 "appare come un vero rivolgimento", collocandosi in una zona "in cui la logica proprietaria non viene del tutto cancellata, ma se ne tenta un significativo ridimensionamento". ${ }^{8}$ Si possono dunque capire le speranze da essa suscitate in chi covava progetti di una rivoluzione sociale.

\footnotetext{
6 Wolin allude alle riforme adottate dopo il colpo di stato del 404, che ridimensionano il potere dell'assemblea, a favore di nomoteti e tribunali.

7 Wolin 1994: 32. Cfr. anche p. 35: “Constitutionalism might be defined as the theory of how best to restrain the politics of democracy while ensuring the predominance of the social groups and classes represented by the "best men"”.

8 S. Rodotà, Il terribile diritto. Studi sulla proprietà privata e i beni comuni, il Mulino, Bologna 2013, pp. 332-33.
} 
Una analoga "impronta sociale" caratterizza la Costituzione brasiliana del 1988, che non solo include un ricco e dettagliato catalogo di diritti sociali, ma alcuni articoli innovativi che vincolano i governi a stanziare una certa percentuale del bilancio pubblico per la loro soddisfazione (penso in particolare agli articoli 198 e 212 sul diritto alla salute e all'istruzione). In Italia, Luigi Ferrajoli ha in più di un'occasione indicato come un modello la Costituzione brasiliana e ha difeso, più in generale, la costituzionalizzazione di vincoli sociali di bilancio, come strumento per "prendere sul serio" una categoria di diritti la cui garanzia presenta difficoltà maggiori di quelle previste per i diritti politici, civili e di libertà. ${ }^{9}$

2. Oggi il modello sociale proposto dalla Costituzione italiana, come da quella brasiliana, è sotto attacco. Nel caso dell'Italia, l'impianto stesso della costituzione del 1948 è stato alterato dall'introduzione del principio dell'“equilibrio di bilancio" nella costituzione, attuata nel 2012 su pressione delle istituzioni europee. Un principio invocato da alcuni - e osteggiato da altri - come una super-norma costituzionale, destinata a prevalere su tutte le altre, impedendo, o per lo meno ostacolando, politiche keynesiane di spesa in deficit. ${ }^{10}$ Se questa fosse l'unica interpretazione possibile, dovremmo concludere - seguendo Wolin - che la Costituzione italiana ha finito col diventare una "camicia di forza" per la democrazia e un serio ostacolo alla possibilità di un reale cambiamento sociale. ${ }^{11}$

Di "camicia di forza" e "pilota automatico" parla, d'altronde, anche chi critica la costituzionalizzazione di vincoli sociali di bilancio simili a quelli previsti in Brasile. Secondo Augustín Menéndez, ad esempio, "El carácter absolutamente infundado de la creencia en el automatismo de las reglas fiscales vale no sólo para las reglas fiscales que fijan topes cuantitativos al déficit y a la deuda pública, sino también para las reglas que prescriben 'suelos', 'porcentajes mínimos' del gasto público o del PIB que hayan de destinarse a determinados gastos (por ejemplos, gastos sociales en su conjunto, educativos, etc.). La inscripción en el 'mármol' constitucional de reglas de este tipo implica también sujetar la politica fiscal al piloto automático, aunque en este caso se trate de un piloto automático orientado a hacer efectivos los objetivos

\footnotetext{
9 Cfr., da ultimo, L. Ferrajoli, Manifesto per l'uguaglianza, Laterza, Roma-Bari 2018, p. 101.

10 Tra coloro che hanno criticato la modifica costituzionale, interpretandola come la costituzionalizzazione delle politiche economiche neo-liberali, cfr. G. Ferrara, Regressione costituzionale, in "Costituzionalismo.it", 18 aprile 2012 e O. Chessa, Pareggio strutturale di bilancio, keynesismo e unione monetaria, "Quaderni costituzionali” XXXVI, 3, 2016, pp. 455-484. Tra gli estimatori, i senatori del PD che il 9 giugno 2015 hanno presentato un disegno di legge in cui si sostiene che "i diritti costituzionali a prestazioni" previsti nella prima parte della Costituzione andrebbero, dopo la riforma, considerati "non assoluti, ma relativi, da graduare nella misura e nel tempo del loro soddisfacimento in modo tale da risultare in equilibrio rispetto agli altri valori costituzionali protetti dall'art. 81 della Costituzione". Cfr. V. Giacché, Costituzione italiana contro trattati europei. Il conflitto inevitabile, Imprimatur, Reggio Emilia 2015, pp. 73-74.

11 In realtà, molti tra i critici dell'inserimento in Costituzione del pareggio di bilancio osservano che la nuova formulazione dell'art. 81 non è tanto restrittiva da impedire politiche keynesiane. E, in ogni caso, invitano a interpretazioni "costituzionalmente orientate" di tale principio, che dovrà pur sempre essere subordinato alla garanzia dei diritti sociali. Cfr. M. Luciani, Costituzione, bilancio, diritti e doveri dei cittadini, "Astrid. Rassegna", 3, 2013; L. Carlassare, Diritti di prestazione e vincoli di bilancio, "Costituzionalismo.it", 3, 2015: F. Pallante, Il problema costituzionale dell'attuazione dei diritti sociali, "DESC”, 1,1 2018.
} 
del Estado social y democratico de Derecho". ${ }^{12}$ Ma è davvero così? E, in ogni caso, possiamo mettere sullo stesso piano i vincoli "anti-sociali" recentemente introdotti nelle costituzioni italiana e spagnola e quelli "sociali" della Costituzione brasiliana del $1988 ?$

Per provare a rispondere a questi interrogativi, è bene tornare sulla questione più generale del rapporto tra democrazia e costituzione, chiedendosi quanti, e che genere, di vincoli può sopportare la democrazia, se vuole continuare ad essere un veicolo di cambiamento sociale. Fino a che punto le politiche economiche possono essere pre-determinate da disposizioni costituzionali? Quanto spazio deve lasciare la costituzione alla libera progettualità politica? Quanto ampia deve essere la "sfera dell'indecidibile"?"13

Escludendo approcci di tipo giusnaturalistico, il problema del rapporto tra democrazia e costituzione può essere affrontato essenzialmente in due modi. Il primo consiste nel giustificare i diritti fondamentali a partire dal principio democratico della sovranità popolare. È una strategia che risale ai padri fondatori della repubblica degli Stati Uniti d'America ai quali appariva evidente che la fonte di ogni legittimo potere - a partire dal potere costituente - fosse il popolo. ${ }^{14}$ In quest'ottica lo stesso controllo di costituzionalità delle leggi non assume una valenza anti-democratica, o "contro-maggioritaria": le norme costituzionali che i giudici sono tenuti ad applicare, infatti, non esprimono altro che la "volontà del popolo", di fronte alla quale la "volontà del legislativo", manifestatasi nelle leggi, passa in secondo piano. ${ }^{15}$

Una simile strategia solleva, tuttavia, una serie di - ben noti - interrogativi. Perché la volontà del popolo solennemente espressasi nel momento costituente dovrebbe contare di più di quella dalle generazioni successive? Perché il passato dovrebbe vincolare il presente al punto da "legare le mani" a chi, oggi, potrebbe non riconoscersi più nelle scelte operate dai costituenti e vorrebbe modificare, senza troppe difficoltà, le norme costituzionali? ${ }^{16}$

Dietro simili interrogativi c'è l'idea che le costituzioni traggano la loro legittimità dal fatto di essere state adottate "democraticamente" e di godere del consenso maggioritario, se non addirittura unanime, dei cittadini. Ma non è affatto scontato che il fondamento assiologico del costituzionalismo vada cercato nel principio del consenso. In società pluraliste e conflittuali

12 A.J. Menéndez, Constitución o camisa de fuerza? De las nuevas reglas fiscale al 'Estado amortizador', "Teoria politica”, n.s., Annali V, 2015, pp. 214-15.

13 Cfr. M. Bovero, Che cosa non è decidibile. Cinque regioni del coto vedado, ”DESC”, n. 1, vol. 1, jul.-dez. 2018, pp. 130-141. Ho affrontato in modo più approfondito questi interrogativi nel quinto capitolo di In nome del popolo. Il problema democratico, Laterza, Roma-Bari 2011 (tr. spagnola En nombre del pueblo. El problema democrático, Marcial Pons, Madrid 2013.

14 A. Hamilton, J. Madison, J. Jay, The Federalist Papers, edited by L Goldman, Oxford University Press, Oxford 2008 , n. 49.

15 Questa tesi è stata ripresa da B. Ackerman, per il quale gli interventi della Corte Suprema sono «democratici» in quanto consistono nella difesa delle decisioni assunte dal popolo nella fase costituente. Cfr. B. Ackerman, We the people, vol. I, Foundations, The Belknap Press of Harvard University Press, Cambridge (Mass.)-London 1991, p 10.

16 Sono le obiezioni contro il costituzionalismo rigido avanzate da J. Waldron in Law and Disagreement, Oxford University Press, Oxford 1999, e riprese in Italia in particolare da Anna Pintore. In questi dibattiti non sempre si tiene conto del fatto che la rigidità delle costituzioni è relativa e variabile: accanto a norme (o principi) sottratti alla revisione costituzionale, vi sono in genere norme modificabili con procedure aggravate. 
come quelle moderne, la costituzione andrebbe piuttosto intesa come un "patto di convivenza" che trova la sua ragion d'essere non nella presunta comunanza di storia e valori di un popolo, ma nell'esigenza di far coesistere pacificamente una molteplicità di opinioni e identità. D'altronde, se davvero le costituzioni traessero la loro legittimità dal consenso, e da questo dipendessero per la loro tenuta, poggerebbero su un fondamento assai fragile, oltre che controverso. Come ha notato in più di un'occasione Luigi Ferrajoli,

azzardato sarebbe supporre che intorno ai valori stabiliti dalle carte costituzionali, inclusi i diritti più elementari, sia esistito, e perfino che esista tuttora, un consenso maggioritario. Un referendum a favore della libertà di coscienza o delle garanzie penali e processuali che si fosse svolto ai tempi di Beccaria o della rivoluzione francese non avrebbe certo avuto il consenso, non diciamo della maggioranza ma neppure di una significativa minoranza. Perfino oggi dovremmo temere, nelle nostre stesse democrazie, una votazione popolare sui diritti sociali o sulla pena di morte17

Una seconda - più promettente - strategia per giustificare l'esistenza di una sfera dell'indecidibile consiste allora nel rovesciare i termini del problema, assumendo che non sia la democrazia a giustificare la costituzione, ma i diritti costituzionali a fungere da presupposto della democrazia.

Le stesse teorie procedurali della democrazia riconoscono, solitamente, che la protezione costituzionale dei diritti politici e di alcuni diritti di libertà è una condizione necessaria perché un sistema possa essere qualificato come democratico. Bobbio sostiene, ad esempio, che il riconoscimento costituzionale dei diritti di libertà rappresenta "il presupposto necessario per il corretto funzionamento degli stessi meccanismi prevalentemente procedurali che caratterizzano un regime democratico". ${ }^{18}$ Michelangelo Bovero ha invitato a intravedere nelle «quattro libertà dei moderni» (libertà personale, di espressione, di riunione, di associazione) le precondizioni liberali della democrazia, e in alcuni diritti sociali, come il diritto alla sussistenza e all'istruzione, le precondizioni sociali che rendono effettive tali libertà. ${ }^{19}$ Luigi Ferrajoli ha sostenuto, a sua volta, l'esistenza di un nesso indissolubile tra la sovranità popolare e ciascuna delle due classi dei diritti da lui denominati "primari": i diritti di libertà e i diritti sociali. Se è vero, infatti, che la volontà popolare "si esprime [...] autenticamente solo se può esprimersi liberamente", attraverso l'esercizio della libertà di espressione, di stampa, di riunione, di associazione, tali libertà sono effettive solo in quanto siano garantite anche le aspettative positive, di pubbliche prestazioni, in cui consistono i diritti socialli. ${ }^{20} \mathrm{Si}$ pensi, in particolare, all'importanza di garantire il diritto all'istruzione per promuovere l'alfabetizzazione politica dei cittadini-elettori.

17 L. Ferrajoli, Principia iuris cit., vol. II, p. 52 (un passo analogo si trova in Diritti fondamentali, p. 344).

18 N. Bobbio, Il futuro della democrazia cit., pp. 6-7.

19 Cfr. M. Bovero, Contro il governo dei peggiori. Una grammatica della democrazia, Laterza, Roma-Bari 2000 , pp. 38-41.

20 L. Ferrajoli, Principia iuris. Teoria del diritto e della democrazia, Laterza, Roma-Bari 2007, vol. II, Teoria della democrazia, p. 7. 
A Ferrajoli è stato obiettato di avere indebitamente ampliato la "sfera del non decidibile", suddividendola nei due emisferi del "non decidibile che" (i diritti di libertà, tutelati da divieti di lesione) e del "non decidibile che non" (i diritti sociali, corrispondenti a obblighi di prestazione a carico delle istituzioni pubbliche). ${ }^{21}$ I critici non negano la necessità di una protezione costituzionale dei diritti politici e di libertà, intesi come condizioni e pre-condizioni della democrazia, ma considerano un'indebita invasione di campo l'inserimento nella "sfera dell'indecidibile" dei diritti sociali, che sembrano avere a che fare più con il contenuto (uno dei possibili contenuti) della democrazia, che con i suoi presupposti. ${ }^{22}$

Vorrei qui sostenere, contro questa tesi, che tra il principio dell'eguaglianza politica - su cui si fonda la democrazia - e quello dell'eguaglianza economico-sociale esiste un nesso difficile da negare. Tale nesso era bene presente fin dall'antichità. Nell'Atene democratica i cittadini che ricoprivano la carica di giurati o di membri del Consiglio, e di alcune altre magistrature, erano ricompensati con un'indennità, chiamata misthos. Introdotto da Pericle nel 462, il misthos viene abolito dagli autori del colpo di stato oligarchico del 411, quindi prontamente ristabilito dai democratici ed esteso anche ai partecipanti all'assemblea. La ragione per cui l'indennità di carica era strettamente associata alla forma di governo democratica era che consentiva di partecipare alla vita politica anche ai poveri, che altrimenti sarebbero risultati esclusi. ${ }^{23}$ Una logica analoga, in fondo, ispira 1'art. 3 della Costituzione italiana, che attribuisce alla Repubblica il compito di rimuovere gli "ostacoli di ordine economico e sociale" che impediscono non solo il "pieno sviluppo della persona umana", ma l'“effettiva partecipazione di tutti i lavoratori all'organizzazione politica, economica e sociale del Paese".

Che dire invece dei vincoli "anti-sociali" che sono stati incorporati nelle costituzioni italiana e spagnola? Possono anch'essi essere interpretati - e giustificati - in nome della democrazia? Si tratta, evidentemente, di una domanda retorica. Ci troviamo in questo caso di fronte a prescrizioni che non solo riducono il novero delle politiche possibili (al pari dei vincoli di natura sociale), ma stravolgono la gerarchia dei diritti tracciata dal costituzionalismo del ventesimo secolo, per affermare il primato dei diritti patrimoniali (come i diritti dei creditori di paesi con forte debito pubblico) sui diritti fondamentali. ${ }^{24}$ Simili vincoli vengono in genere giustificati con argomenti di natura tecnica: si tratterebbe di salvaguardare i principi della corretta economia, a partire dal

21 L. Ferrajoli, Principia iuris, cit., vol. I, Teoria del diritto, p. 822.

22 Cfr. in particolare A. Pintore, Diritti insaziabili, in L. Ferrajoli, Diritti fondamentali, a cura di E. Vitale, Laterza, Roma-Bari 2001, pp. 179-200 e Ead., I diritti della democrazia, Laterza, Roma-Bari 2003.

23 Mi permetto di rimandare a V. Pazé, La democracia de los antiguos, la democracia de los modernos, in Democracia o postdemocracia? Problemas de la representación politica en las democracias contemporáneas, a cura di Luis Salazar Carrión, Fontamara, Città del Messico 2014, pp. 31-46.

24 Sulla distinzione tra diritti fondamentali e patrimoniali, e sul rapporto di subordinazione dei secondi ai primi, cfr. ancora L. Ferrajoli, Principia iuris cit., vol. I, pp. 724 e sg. 
necessario equilibrio tra entrate e uscite. Ma non è difficile scorgere dietro di essi - bocciati da economisti del calibro di Stiglitz e Krugman - la determinazione a "mettere in sicurezza" non la democrazia e i suoi presupposti, ma l'attuale assetto del capitalismo finanziario.

3. Se non è particolarmente difficile giustificare, sul piano dei principi, la costituzionalizzazione dei diritti sociali, intendendoli come pre-condizioni della democrazia, resta il problema della loro effettività. L'introduzione di vincoli di bilancio simili a quelli previsti dalla Costituzione brasiliana è uno strumento efficace per costringere il legislatore a tener fede al dettato costituzionale? Di fatto, sono molti i modi in cui tali vincoli possono essere ridimensionati o elusi: il ricorso a interpretazioni "creative" delle norme che li dispongono, la previsione di eccezioni giustificate invocando vincoli finanziari inaggirabili, l'inadeguatezza dei controlli giurisdizionali, la limitata effettività delle sanzioni, l'introduzione di emendamenti costituzionali. ${ }^{25} \mathrm{Il}$ problema di fondo - ha sostenuto Humberto Ávila - è che "as vinculações sociais não possuem força vinculante em si, mas força vinculante em razão das atividades de interpretação e de aplicação". ${ }^{26}$ Lo stesso studioso insiste, tuttavia, sul significato di simili vincoli: "Mas mesmo que as vinculações sociais não sejam efetivas em alto grau, tendo muitas vezes um caráter meramente simbólico, como ocorre no Brasil, ainda assim elas são importantes para criar a cultura da socialidade e impor limites à atuação estatal. Ė evidente que o melhor é sempre um limite rígido [...]. Mas entre ter um limite débil e nenhum limite, é obviamente melhor um limite débil, desde que as exceções abertas às regras sejam efetivamente extraordinárias e objetivamente comprovadas, pois se assim não for, na verdade não haverá limite algum à atividade financeira do Estado", ${ }^{27}$

Concludo osservando che non bisogna nutrire esagerate aspettative nei confronti delle potenzialità "rivoluzionarie" dei vincoli sociali di bilancio, e dei giudici incaricati di farli rispettare. Se la costituzionalizzazione di vincoli di bilancio "costringe" opportunamente la politica a non dimenticarsi dei diritti sociali, il legislatore continua ad avere ampi margini di discrezionalità non solo nel decidere quanto stanziare, al di là della percentuale minima stabilito, e quali politiche adottare, ma anche nell'intervenire sul lato delle entrate, attraverso politiche fiscali più o meno progressive.

Riprendendo le considerazioni di Bobbio con cui ho iniziato, è inoltre banale osservare che la costituzionalizzazione del "diritto alla rivoluzione" non conduce da nessuna parte in assenza di soggetti politici collettivi, in grado di elaborare progetti credibili di trasformazione sociale.

\footnotetext{
25 H. Ávila, Os vínculos sociais na Constituição brasileira, “Teoria politica”, n.s., Annali V, 2015, pp. 221-233.

26 Ivi, p. 232.

27 Ivi, p. 233.
} 


\section{Bibliografia}

ACKERMAN, B. We the people. Vol. I, Foundations. Cambridge-London: The Belknap Press of Harvard University Press, 1991.

ÁVILA, H. Os vínculos sociais na Constituição brasileira. Teoria politica, Annali V, 2015, p. 221-233.

BOBBIO, N. Il futuro della democrazia. Torino: Einaudi, 1984.

L'età dei diritti. Torino: Einaudi, 1990.

La rivoluzione come movimento e mutamento. In: BOVERO, M. (org.) Teoria generale della politica.

Torino: Einaudi, 1999.

BOVERO, M. Che cosa è non decidibile: cinque regioni del coto vedado. Revista DESC - Direito, Economia e Sociedade Contemporânea, v. 1, n. 1, 2018, p. 129-141. DOI 10.33389/desc.v1n1.2018.p129-141.

Contro il governo dei peggiori. Una grammatica della democrazia. Roma-Bari: Laterza, 2000.

CARLASSARE, L. Diritti di prestazione e vincoli di bilancio. Costituzionalismo.it, f. 3, 2015.

CHESSA, O. Pareggio strutturale di bilancio, keynesismo e unione monetaria. Quaderni costituzionali, a. XXXVI, n. 3, 2016. DOI 10.1439/84265.

FERRAJOLI, L. Manifesto per l’uguaglianza. Roma-Bari: Laterza, 2018.

Principia iuris. Teoria del diritto e della democrazia. Roma-Bari: Laterza, 2007.

FERRARA, G. Regressione costituzionale. Costituzionalismo.it, 18 aprile 2012.

GIACCHÉ, V. Costituzione italiana contro trattati europei. Il conflitto inevitabile. Reggio Emilia: Imprimatur, 2015.

HAMilton, A.; Madison, J.; Jay, J. The Federalist Papers. Oxford: Oxford University Press, 2008.

LUCIANI, M. Costituzione, bilancio, diritti e doveri dei cittadini. Astrid Rassegna, n. 3, 2013.

MENÉNDEZ, A.J. ¿Constitución o camisa de fuerza? De las nuevas reglas fiscale al "Estado amortizador”. Teoria politica, Annali V, 2015, p. 189-219.

PALLANTE, F. Il problema costituzionale dell'attuazione dei diritti sociali. Revista DESC - Direito, Economia e Sociedade Contemporânea, v.1, n.1, 2018, p. 70-82. DOI 10.33389/desc.v1n1.2018.p70-82.

PAZĖ, V. In nome del popolo. Il problema democratico. Roma-Bari: Laterza, 2011 (tr. spagnola En nombre del pueblo. El problema democrático. Madrid: Marcial Pons, 2013).

La democracia de los antiguos, la democracia de los modernos. In: CARRIÓN, L.S. (org.). Democracia o postdemocracia? Problemas de la representación política en las democracias contemporáneas. Città del Messico: Fontamara, 2014, pp. 31-46.

PINTORE, A. Diritti insaziabili. In: FERRAJOLI, L. Diritti fondamentali. Roma-Bari: Laterza, 2001, p. 179-200. I diritti della democrazia. Roma-Bari: Laterza, 2003.

RODOTÀ, S. Il terribile diritto. Studi sulla proprietà privata e i beni comuni. Bologna: Il Mulino, 2013.

WALDRON, J. Law and Disagreement. Oxford: Oxford University Press, 1999.

WOLIN, S. Norm and Form: The Constitutionalizing of Democracy. In: EUBEN, J.P.; WALLACH, J.R.; OBER, J. (eds.). Athenian Political Thought and the Reconstruction of American Democracy. Ithaca-London: Cornell University Press, 1994. 
Valentina Pazé

Professora associada de Filosofia Política junto ao Departamento de Cultura, Política e Sociedade da Universidade de Turim. Autora, dentre outros trabalhos, de: Il concetto di comunità nella filosofia politica contemporanea (Laterza, 2002), Comunitarismo (Laterza, 2004), In nome del popolo (Laterza, 2011), Cittadini senza politica. Politica senza cittadini (EGA, 2016). 